\title{
Le réseau de la terreur : une hypothèse à revisiter
}

\section{Michel Wieviorka}

\section{(2) OpenEdition \\ Journals}

Édition électronique

URL : http://journals.openedition.org/conflits/127

DOI : $10.4000 /$ conflits. 127

ISSN : $1777-5345$

Éditeur :

CCLS - Centre d'études sur les conflits lilberté et sécurité, L'Harmattan

Édition imprimée

Date de publication : 17 janvier 1991

ISSN : 1157-996X

Référence électronique

Michel Wieviorka, «Le réseau de la terreur : une hypothèse à revisiter », Cultures \& Conflits [En ligne],

04 | hiver 1991, mis en ligne le 31 décembre 2002, consulté le 30 mars 2021. URL : http://

journals.openedition.org/conflits/127 ; DOI : https://doi.org/10.4000/conflits. 127

Ce document a été généré automatiquement le 30 mars 2021.

Creative Commons License 


\title{
Le réseau de la terreur : une hypothèse à revisiter
}

\author{
Michel Wieviorka
}

Une hypothèse controversée

Au début des années 80 , le terrorisme fut l'objet d'une interprétation qui connut un succès considérable : qu'il s'agisse de ses variantes d'extrême gauche, ou d'inspiration nationaliste, qu'il soit interne ou international, on le comprenait d'autant mieux qu'on faisait l'hypothèse d'un réseau de la terreur, orchestré depuis l'Union soviétique et ses pays satellites ${ }^{1}$. Le livre de Claire Sterling, The Terror Network ${ }^{2}$ obtint un écho impressionnant, il fut traduit dans le monde entier, et de nombreux ouvrages

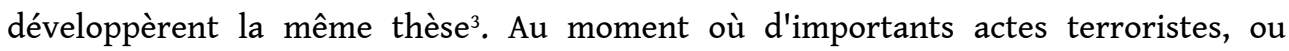
assimilables, procédaient de l'islam radical, dans une conjoncture où l'Iran, la Syrie et l'Irak commençaient à apparaître comme les principaux " sponsors " du terrorisme international, l'idée paradoxale d'un réseau activé depuis Moscou séduisait non seulement le gouvernement américain, mais aussi de larges pans de l'opinion publique, aux Etats-Unis comme ailleurs. Elle soulevait pourtant de sérieuses réserves, qui s'orientèrent autour de trois axes principaux. Le premier renversait tout simplement le raisonnement de Claire Sterling pour affirmer que le seul vrai réseau de la terreur ${ }^{\wedge}$ the real Terror network ^était celui déployé dans le monde par les Etats-Unis et la CIA, ainsi que par Israël ${ }^{4}$. Le second consistait à mettre en cause l'image même d'un réseau, à souligner le manque de preuves à l'appui de cette image, et à postuler une plus grande hétérogénéité du phénomène terroriste. Au sein de la CIA, et du Département d'état américain, la thèse de Claire Sterling n'a jamais été véritablement acceptée ${ }^{5}$, et Bob Woodward, dans son ouvrage sur la CIA, a même raconté, quelques années plus tard, comment la journaliste aurait été en fait manipulée par les services secrets américains(5). Enfin, une troisième famille de critiques, renforçant la précédente, demandait qu'on analyse le terrorisme par le bas, à partir de ses sources sociales, culturelles et politiques, et non par le haut, à partir de l'idée d'un complot ou d'une vaste manipulation mise en oeuvre par l'URSS et les pays situés dans sa zone d'inf1uence ${ }^{6}$. Dans cette perspective, l'hypothèse d'un réseau mis en place par le 
Kremlin pouvait au mieux laisser la place à l'idée de rencontres entre des acteurs terroristes en quête de soutien logistique, et, de façon plus ou moins occasionnelle, des responsables de pays communistes intéressés à les utiliser au coup par coup, le cas échéant. Le rôle de ces pays apparaissait plutôt en bout de course, non pas comme un facteur explicatif du terrorisme, mais comme le point d'aboutissement de dérives, de processus dans lesquels des acteurs trouvaient en fin de parcours les repères politiques et l'aide pratique que tel ou tel état voulait bien leur fournir. Encore faut-il préciser que dans cette perspective, les acteurs en question ne versaient pas dans une totale hétéronomie ou subordination, qu'ils ne devenaient pas de simples pions à la solde d'un régime communiste et étaient capables de maintenir des projets qui leur étaient propres. La thèse du réseau de la terreur s'est trouvée non seulement contestée, mais aussi considérablement affaiblie à partir des années 83-84. Elle fut sinon délaissée, du moins minimisée, y compris par l'administration américaine, en même temps que d'autres propositions, beaucoup moins idéologiques, mettaient l'accent sur le rôle de la Libye, de l'Irak, de la Syrie et de l'Iran ${ }^{7}$, et sur l'autonomie relative de certains groupes radicaux.

3 Puis vinrent la glasnost et la perestroïka, la destruction du mur de Berlin, l'indépendance des anciens états satellites de l'empire soviétique. Ce formidable chambardement a profondément affecté non seulement le phénomène terroriste, mais aussi nos connaissances, nous invitant à revisiter la thèse du Terror Network. L'Est entre passé Au niveau des déclarations officielles et de la presse, tout comme dans des textes plus élaborés, par exemple sous la signature de Viktor Vitiuk, l'Union soviétique s'est généralement défendue d'orchestrer ou même d'inspirer l'ensemble des expériences qualifiées de terroristes dans le monde occidental. Le terrorisme d'extrême gauche, d'inspiration maoïste, ou foquiste, y a été le plus souvent présenté comme une perversion du marxisme et du léninisme, et si, à l'Ouest, il était de plus en plus couramment perçu comme le contraire de la démocratie, à l'Est, il fut constamment critiqué comme étant le contraire de la Révolution. Les groupes d'extrême gauche y étaient décrits sans concession, dénoncés pour leur violence spontanéiste et petitebourgeoise qui renforçait de fait le capitalisme et l'impérialisme américain. Ce n'est que là où la violence pouvait incarner une lutte de libération nationale, et anti-impérialiste, qu'elle pouvait éventuellement trouver grâce et soutien du côté de l'Union soviétique. Mais, même là, l'idée d'une reconnaissance et d'une aide directe au terrorisme mérite d'être nuancée. C'est ainsi, par exemple, que des documents saisis dans des locaux palestiniens par l'armée israélienne lors de l'opération Paix en Galilée au Liban, en 1982, et diffusés sous la forme d'un livre de propagande par l'état d'Israël, indiquent clairement que si des organisations palestiniennes ont bénéficié d'un soutien logistique ou d'entraînements militaires dans plusieurs pays communistes, elles n'en ont pas pour autant été nécessairement encouragées au terrorisme proprement dit. Le script d'une rencontre entre Arafat, Gromiko et Ponomarev, est de ce point de vue particulièrement édifiant, et à la limite, on peut s'étonner que les services israéliens de propagande, si soucieux de montrer le contraire, aient choisi de le publier dans ce Livre ${ }^{8}$. Mais ne confondons pas les déclarations officielles ou publiques avec la réalité. En fait, dans de nombreuses circonstances, l'attitude de Moscou vis-à-vis d'organisations pratiquant la lutte armée a été changeante ou ambivalente, et déterminée non pas tant par des positions idéologiques, mais par des préoccupations de géopolitique dans lesquelles la notion de terrorisme, en tant que telle, ne constituait pas une variable déterminante. Le régime soviétique, ou ses alliés, ont agi en fonction d'intérêts internationaux qui l'ont 
fait soutenir, ou non, l'action armée de groupes et mouvements qualifiés, selon la conjoncture, de résistants lorsqu'il s'agissait de les aider, et de terroristes sinon. Ainsi, les Tamouls au Sri Lanka ont été nettement appuyés jusqu'en 1987, puis qualifiés de plus en plus souvent de terroristes à partir de cette époque.

4 Avec l'indépendance des pays satellites de Moscou, un premier constat s'impose : la densité des relations de ces pays avec des états et des groupes terroristes a été considérable, et bien dans le sens de la thèse du Terror Network. Rien n'indique, certes, qu'il y ait eu réellement un unique réseau structuré, organisé. Mais tout converge pour suggérer qu'une multiplicité de liens ont existé entre les uns et les autres. L'Allemagne de l'Est a apporté plus qu'un refuge aux activistes de la Fraction Armée Rouge, qui y ont bénéficié, on le sait maintenant, d'importants appuis. La Pologne a accueilli, en particulier, le groupe Abou Nidal, qui disposait à Varsovie, comme le lobby juif AIPAC l'a dénoncé dès 1987 aux Etats-Unis, d'une véritable antenne permanente, à peine camouflée sous une vague raison sociale. La Tchécoslovaquie a fourni à des groupes terroristes, directement ou indirectement, d'impressionnantes quantités d'un explosif, le Semtex, particulièrement prisé par les terroristes. Le produit présentait en effet la caractéristique de ne pas être détectable à l'odeur. Il a servi à faire exploser le Boeing 747 de la Pan-Am au dessus de Lockerbie (21 décembre 1988), et, pour la seule année 1989, les services de sécurité du Royaume-Uni estiment à 200 le nombre des attentats commis avec lui en Irlande du Nord. Le 22 mars 1990, le président tchécoslovaque, Vaclav Havel, a révélé, à l'occasion d'une conférence de presse, que son pays avait exporté mille tonnes de Semtex vers la Libye. Il a également annoncé une modification de la préparation du Semtex, désormais marqué par une odeur chimique aisément détectable; et aujourd'hui, cet explosif, largement commercialisé, concurrence sérieusement, par ses qualités et son prix, les produits présents sur le marché occidental. Bref, révélation après révélation, il se précise et se confirme que toutes sortes de réseaux ont tissé des relations, dont on ignore jusqu'à quel point elles ont pu être interconnectées, entre des entreprises travaillant dans le secteur de l'armement, des centres de pouvoir plus ou moins discrets, des acteurs appartenant à divers appareils des régimes de l'Est, et des organisations terroristes en relation directe avec eux, ou trouvant dans des états comme la Libye un éventuel intermédiaire, quand ces états n'agissaient pas directement pour leur compte, sur un mode terroriste. Les liens sont même parfois étonnants, comme par exemple ceux qui ont existé entre la Bulgarie et l'extrême droite turque au milieu des années 80 .

5 Il faut donc reconnaitre que, si elle s'appuyait sur des affirmations contestables, sur des informations émanant de personnages douteux, ou puisées à des sources non vérifiables, la thèse du réseau de la terreur s'avère plus pertinente que ce que ses critiques en ont dit. Elle a souvent été développée sur un mode outrancier, très idéologique, et présentée abusivement comme une explication générale du phénomène terroriste. Mais elle a eu le mérite, malgré ses défauts, de souligner le rôle majeur de régimes sans qui le phénomène n'aurait assurément pas connu l'extension qui fut la sienne dans les années 70 et 80 . et présent une confirmation a contrario de ce qui vient d'être signalé est dans l'évolution du terrorisme, depuis la dislocation de l'empire soviétique et la mutation des régimes qui le constituaient. Si le terrorisme n'a pas disparu, s'il est toujours susceptible de frapper ça et là, force est de constater qu'il occupe la Une de nos journaux bien moins que par le passé, qu'il a décliné dans la hiérarchie des préoccupations majeures de l'opinion publique dans de nombreux pays. Ce changement n'est évidemment pas réductible aux seules mutations de l'ancien 
empire soviétique, mais il y doit beaucoup, ne serait-ce que parce que les pays concernés non seulement ont cessé d'apporter leur soutien, direct ou indirect, à de nombreux acteurs du terrorisme, mais aussi parce qu'ils s'intègrent de plus en plus à l'action internationale contre le terrorisme. Ces pays n'ont jamais été entièrement étrangers aux efforts internationaux de coopération antiterroriste. Ils ont notamment été partie prenante de la lutte contre la piraterie aérienne, qui faut-il le préciser, était susceptible de les affecter eux aussi, comme on l'a vu à plusieurs reprises avec les détournements d'avion opérés par des dissidents, des nationalistes lituaniens ou des juifs " refuzniks " tentant de quitter l'URSS. En 1973, les Etats-Unis et Cuba ont même signé un accord où les deux parties s'engageaient à poursuivre ou extrader les auteurs de tels piratages, et à restituer tout appareil détourné. Mais ce n'est qu'avec le tournant de la perestroïka qu'une collaboration s'est largement mise en place entre l'Ouest et l'Est. Un premier signe en fut donné en 1987, lorsque la délégation soviétique à la Conférence sur la Sécurité et la Coopération en Europe a proposé de réunir un colloque d'experts sur la prévention du terrorisme international. En janvier 1989, un accord était signé par l'Union soviétique, les Etats-Unis, le Canada et tous les pays européens, incluant une disposition où le terrorisme était condamné comme injustifiable en toutes circonstances. A la même époque, la " Literaturnaïa Gazeta " organisait une très importante table ronde, où des experts occidentaux ont $\mathrm{pu}$ rencontrer leurs homologues soviétiques. En conclusion de cette rencontre, il fut demandé que soit mise sur pied une " task force " américano-soviétique, susceptible de produire des études en commun, et d'élaborer des propositions en matière d'action antiterroriste. Déjà annoncé en décembre 1985 par le vote du bloc soviétique, aux Nations unies, de résolutions condamnant le terrorisme, le changement, radical, a été confirmé, très concrètement, par l'amorce de relations bilatérales entre l'Union soviétique et plusieurs pays occidentaux, en particulier en matière d'échanges d'informations sur des individus et des groupes, d'assistance technique et de réflexion conjointe en matière de droit international. Et avant même que les relations diplomatiques soient rétablies entre l'URSS et Israël, on a pu constater, à l'occasion d'un détournement d'avion soviétique qui s'est posé sur l'aéroport de Tel Aviv en décembre 1988, qu'une coopération antiterroriste pouvait se nouer entre les deux états. Comme le montrent aussi les travaux pionniers de Martha Crenshaw et Galia Golan', l'URSS et ses anciens satellites ont donc cessé d'animer le, ou plutôt les réseaux de la terreur, pour devenir partie prenante de réseaux antiterroristes. Encore faut-il ne pas faire preuve de naïveté excessive. Comme le signale Galia Golan, le soutien du Kremlin, à la fin des années 80, est demeuré bien réel à l'égard d'organisations de lutte armée, en particulier aux Philippines et au Pakistan ${ }^{10}$, et si la tendance générale est bien celle qui vient d'être dessinée, d'importants écarts demeurent possibles. Le rapprochement des positions de l'Est et de l'Ouest ne signifie pas partout et en tous temps un alignement absolu, et tout n'est pas réglé, à l'Est, en ce qui concerne la dissolution des appareils qui ont pu ça et là aider des groupes terroristes. Prospective Mais faut-il s'arrêter à cette image de réseaux terroristes affaiblis et même privés de capacité d'action du fait de l'effondrement des régimes totalitaires? Si les opinions occidentales sont moins sensibles au phénomène terroriste, s'il a effectivement été affaibli il n'en a pas pour autant disparu. Et qui plus est, il semble capable de s'en prendre, précisément, aux intérêts des pays de l'ancien bloc communiste. Il y a quelques années à peine, en 1986, circulait une rumeur significative. Quatre diplomates soviétiques ayant été enlevés à Beyrouth-Ouest par un groupe islamiste, dans un contexte où se démultipliaient les 
enlèvements d'Occidentaux, et l'un d'entre eux ayant été assassiné, la réponse aurait été foudroyante : les Soviétiques auraient eux-mêmes kidnappé un parent de Cheikh Fadlala, leader spirituel et politique chute, l'auraient amputé d'un doigt et auraient fait savoir, en faisant parvenir le doigt amputé à la famille, qu'il fallait s'attendre à ce genre de rétorsion. Très vite les trois otages survivants auraient été libérés.

6 Aujourd'hui, les groupes proche-orientaux sont moins regardants s'il s'agit des pays de l'Est, et susceptibles d'exercer à leur encontre un chantage terroriste difficilement concevable deux ou trois ans auparavant. C'est ainsi que le transfert des juifs d'URSS vers Israël, qui emprunte, entre autres canaux, des avions relevant des compagnies d'aviation hongroise et polonaise, a suscité en mars 1990 des menaces vis-à-vis des pays concernés, et que ces menaces ont été suivies, pour la Pologne, d'actes concrets ^ un ressortissant polonais a été blessé à Beyrouth. Il faut donc considérer que le terrorisme, notamment international, n'est pas épuisé définitivement par l'effondrement des régimes communistes, que ses virtualités et sa réalité demeurent, et que des réseaux autres que ceux aboutissant à Moscou perdurent ou se reconstituent, en particulier au Proche-Orient. L'assassinat de Chapour Baktiar à Paris, en 1991, a bien montré, par exemple, que des réseaux sont capables de mettre sur pied des opérations vraisemblablement " sponsorisées " par l'état iranien, en l'occurrence, ou certains secteurs de ses appareils.

7 I1 faut même aller plus loin, et se livrer à une prospective ^ exercice toujours aléatoire, mais qui a le mérite d'envisager des scénarios ayant une certaine vraisemblance. Rien n'interdit de penser en particulier, que la décomposition et l'éclatement de l'ancien empire soviétique puissent se solder par la formation de mouvements terroristes, dont les lieux ne sont pas difficiles à imaginer. Une première virtualité se situe du côté des mouvements nationalistes, dont l'éventuelle violence peut toujours prendre des formes terroristes, en amont ou en aval de processus plus massifs, ou pour les accompagner. $\mathrm{Au}$ nationalisme ouvert et démocratisant qui s'est armé dans les républiques baltes, comment ne pas opposer, en effet, les conduites de haine et de rupture, comme on a pu les observer, notamment au Caucase, avec les violences réciproques des Azéris et des Arméniens, en Bulgarie, à l'encontre des Turcs, en Roumanie, à l'encontre des minorités hongroises de Transylvanie, etc. ? La désintégration du système communiste constitue assurément une condition favorable à l'émergence de mouvements susceptibles de virer au terrorisme et au contre-terrorisme, en particulier dans des régions du monde qui, tels les Balkans, ont en la matière une épaisse tradition historique. Une deuxième virtualité concerne les régions à forte proportion de musulmans, où il ne faut pas exclure des processus de radicalisation islamiste, s'inspirant, jusqu'au terrorisme, de l'islam fondamentaliste tel qu'il s'est déployé notamment au Proche et au Moyen-Orient dans les années 80.

Une troisième hypothèse est celle d'un terrorisme antisémite pogromiste, animé par des doctrinaires tentant de mobiliser des secteurs populistes de la population sur une base nationaliste dure, du type de celle qui incarne en Russie Pamyat. Cette organisation a, dans le passé récent, été fortement surestimée, en Occident mais aussi en Russie, où son impact, jusqu'ici, demeure extrêmement minoritaire. Mais dans la dégradation continue de la société russe, le chaos ou l'autoritarisme qui menacent, cette hypothèse mérite d'être maintenue. Bien d'autres virtualités pourraient être suggérées: instrumentation de groupes sociaux en colère, comme les mineurs de Roumanie, formation de bandes oscillant entre la délinquance et la criminalité 
organisée, d'un côté, et, d'un autre côté, un terrorisme de déstabilisation politique, destiné par exemple à écarter toute reprise en main par l'état de situations où un pouvoir local tente de s'autonomiser, etc. De tels scénarios ne constituent pas une pure fiction. En novembre 1991, par exemple, le Président d'une république musulmane du Caucase rattachée à la Russie, la Tchétchéno-Ingouchie, a menacé Moscou d'actions terroristes visant les centrales nucléaires. Mais n'allons pas trop loin. L'essentiel, ici, est d'admettre que l'ordre communiste, s'il a pu dans le passé inspirer, commanditer ou aider des groupes terroristes, non seulement s'écarte d'une telle orientation, mais aussi laisse la place à des situations où une violence extrême trouve un espace et des conditions favorables. Ce qui nous permet, en fin de compte, de mieux contextualiser la thèse du Terror Network du début des années 80. Cette thèse a mis le doigt sur une réalité incontournable, le rôle des régimes communistes dans l'extension du phénomène terroriste, au fil des années 70 . Elle a aussi permis de construire des raisonnements insistant, au-delà de l'expérience soviétique, sur l'existence de réseaux animés par le haut, " sponsorisant ", à partir d'états ou de certains centres de pouvoir, l'action de groupes ayant besoin de logistique, d'entraînement, de sanctuaires Mais en aucune façon, elle n'a apporté une théorie du terrorisme, elle n'a jamais expliqué comment et pourquoi des acteurs étaient amenés à recourir à cette forme si particulière de violence, et à accepter de s'insérer dans des réseaux et des logiques dont ils étaient souvent, au départ, relativement éloignés. Le phénomène demeure une virtualité permanente, précisément parce qu'il n'a pas besoin, pour jaillir et se développer, de réseaux orchestrés de plus ou moins loin. Mais il est vrai aussi que de tels réseaux lui apportent de quoi assurer une certaine pérennité ou stabilité dans le temps, en même temps que leurs animateurs y trouvent leur compte politique et géopolitique.

\section{NOTES}

1. Cet article reprend certains éléments du rapport Présent et avenir du terrorisme, quelques hypothèses, rédigé pour le Centre international de criminologie comparée de l'Université de Montréal (1991).

2. Claire Sterling, The Terror Network, New York, Holt, Rinehart and Winston : The Reader s Digest Press, 1981

3. Cf. aux Etats-Unis, Ray S. Cline, Yonah Alexander, Terrorism : the soviet connection, New Yorh : Crane Russah, 1984, et en France, par exernple, Edouard Sablier Le fil rouge, Paris : Plon, 1983.

4. Edward Herrnan, The Real Terror Network, Boston : South End Press, 1982. 4. Sur ce point et, plus largement, sur les analyses et les politiques américaines face au terrorisme, cf. Michel Wieviorha, " Defining and implementing foreign Policy : the U.S. Experience in Anti-terrorism ", inThe 1988-1989 Annual on Terrorism, Yonah Alexander, Abraham H. Foxman (eds), Dordrecht, Martinus Nijhoff Pub., 1990, pp. 171-201. 
5. Bob Woodward, Veil, the secret Wars of the CIA, New York : Simon and Schuster, 1987.

6. Cette perspective a animé nos propres trauvaux, cf Michel Wieviorka, Sociétés et terrorisme, Paris, Fayard, 1988.

7. D'où la notion d'état-sponsor appliquée à ces états. Cf. Ray S. Cline et Yonah

Alexander, Terrorism as State sponsored covert Warfare, Fairfax, VA, Hero Boohs, 1986.

8. Cf PLO in Lebanon, Londres : Weidenfeld and Nicolson, 1983.

9. Cf. Martha Crenshaw, Terrorism and international Cooperation, Occasional Papers Series, n 11, Nem York : Institute for East-West Security Studies, 1989 ; Galia Golan, Gorbatchev's, " New Thinking " on Terrorism, New York : Praeger, 1990, et The Soviet Union and national Liberation Movements in the Third World, Londres : Unwin and Hyman, 1988.

10. Galia Golan, Gorbatchev's, op. cit, pp. 80-83.

\section{RÉSUMÉS}

Michel Wieviorka réexamine la thèse du réseau de la terreur manipulé par l'URSS. Cette thèse formulée en général d'une manière outrancière et idéologique par ceux qui l'ont mise à la mode à la fin des années 70, a connu un désintérêt croissant avec l'arrivée sur la scène du terrorisme des pays du Moyen-Orient, puis avec la fin du communisme. A la lueur des informations récentes l'auteur montre que cette thèse s'avère plus pertinente que ses critiques ne l'ont pensé à l'époque, en revanche, si elle décrit assez bien le fonctionnement des - et non du - réseaux de la terreur, elle n'explique pas pourquoi ce type de violence a pu s'enraciner localement et elle ne saurait en aucune manière constituer une théorie du terrorisme.

The author re-examines the hypothesis of a terrorist network manipulated by the USSR. This belief was often presented in an excessive and highly ideological manner at the end of the 70's. Since then, with the rise of Middle-Eastern terrorism and the end of communism, it has lost much of its appeal. However the author shows, in the light of recent data, that the thesis was not as unfounded as was contended by its critics at the time; it describes quite adequately the functioning (or failure) of terror networks, but it fails to show why and how this type of violence found local roots and it doesn't lend itself in any way as a basis for the establishment of a theory of terrorism.

INDEX

Mots-clés : terreur, terrorisme, réseaux transnationaux

\section{AUTEUR}

\section{MICHEL WIEVIORKA}

Directeur d'Etudes à l'Ecole des Hautes en Sciences Sociales. 